\title{
Clinical, Imaging and Pathological Characteristics of Brain Implanted Polylactic Co-Glycolic Acid Polymers Conjugated with Temozolomide
}

\author{
Jill Hicks ${ }^{1^{*}}$, Simon Platt $\mathbf{R}^{1}$, Shannon Holmes $\mathbf{P}^{1}$, Elizabeth Howerth ${ }^{1}$, Allison Haley ${ }^{1}$, Jared Kaplan $\mathbf{M}^{3}$ and Edward Kaplan $\mathbf{J}^{2}$ \\ ${ }^{1}$ Department of Small Animal Medicine and Surgery, College of Veterinary Medicine, 2200 College Station Rd, University of Georgia, Athens, GA 30602, USA \\ ${ }^{2}$ Microspherix LLC, Boca Raton, FL 33428, USA \\ ${ }^{3}$ Department of Biochemistry and Biology, University of Florida, Gainesville, FL 32611, USA
}

*Corresponding author: Jill Hicks, Department of Small Animal Medicine and Surgery, College of Veterinary Medicine, 2200 College Station Rd, University of Georgia, Athens, GA 30602, USA, Tel: 973-303-8966; E-mail: jhicks30@uga.edu

Rec date: Mar 26, 2016; Acc date: Apr 21, 2016; Pub date: Apr 23, 2016

Copyright: ( 2016 Hicks J, et al. This is an open-access article distributed under the terms of the Creative Commons Attribution License, which permits unrestricted use, distribution, and reproduction in any medium, provided the original author and source are credited.

\begin{abstract}
Definitive treatments for primary brain tumours are still sought. Slow-release local chemotherapy may provide a good effect and poly (lactide-co-glycolide) (PLGA) microcylinders could allow this. We evaluated the neurological and histopathological consequences, and MRI visibility, of PLGA microcylinders conjugated with temozolomide and gadolinium implanted in normal canine brains. Eight purpose-bred beagles had cerebral implantation of microcylinders combined with temozolomide and gadolinium. MRI was performed following implantation and 28d later prior to necropsy and brain histopathology. All adverse events were associated with implantation and resolved. Dogs with six microcylinders at 0 and $6.25 \%$ gadolinium had mild inflammation and all other dogs had greater brain inflammation, which increased with higher gadolinium concentrations and microcylinder number. Microcylinders with gadolinium were identifiable on MRI. Brain implantation of PLGA microcylinders conjugated with gadolinium and temozolomide is tolerated in healthy beagles. The lowest gadolinium percentage and microcylinder number should be used if this therapy is pursued.
\end{abstract}

Keywords: Local chemotherapy; Primary brain tumor; Dog; Magnetic resonance imaging; Gadolinium

\begin{abstract}
Abbreviations
AB/PAS: Alcian blue/Periodic Schiff; BBB: Blood Brain Barrier; CD3: T Cell Marker; CD18: Microglia and Macrophage Marker; CD79: B Cell Marker; CNS: Central Nervous System; CSF: Cerebrospinal Fluid; FLAIR: Fluid Attenuation Inversion Recovery; GBM: Glioblastoma Multiforme; GFAP: Glial Fibrillary Acidic Protein; AM: Astrocyte Marker; HE: Hematoxylin and eosin; MPRAGE: Magnetization-Prepared Rapid Acquisition Gradient Echo; MRI: Magnetic Resonance Imaging; PDW: Proton Density Weighted; PLGA: Polylactic Co-glycolic Acid; RBCs: Red Blood Cells; SPACE: Spatial and Chemical Shift Encoded Excitation; T1w: T1 Weighted; T2w: T2 Weighted; $\mathrm{T}^{\star}$ : $\mathrm{T}^{\star}$ Weighted; TMZ: Temozolomide; TNCC: Total Nucleated Cell Count; TruFISP: True Fast Imaging with Steady State Precession; WBCs: White Blood Cells.
\end{abstract}

\section{Background}

Primary brain tumours occur more commonly in dogs than any other domestic species [1] and while their incidence is only reported to range from $14-20 / 100,000$ to up to $3 \%$ of the canine population, [2,3] the effects of brain tumours on both dogs and their families are often devastating. Meningiomas, the most common primary brain tumour of dogs $[4,5]$ have shown good response to more extensive surgical resection [6-8] and both conventional and stereotactic radiation therapy $[9,10]$. Glial tumours arise from the neuroectoderm and most notably include low-grade astrocytomas, oligodendrogliomas, and glioblastoma multiforme, representing $17 \%, 13 \%$ and $3 \%$ of primary canine brain tumours respectively $[5,11,12]$. These tumours have less definitive information regarding their treatment and appear to be less responsive to conventional therapies overall [13].

Glioblastoma multiforme (GBM) is the most common primary malignant brain tumour in humans, and is associated with a poor long-term prognosis despite multimodal therapy [14]. Temozolomide (TMZ) is an imidazotetrazine derivative of the alkylating agent dacarbazine and has become standard of care as part of the multimodal treatment for GBM in humans [15]. Temozolomide treatment led to an improvement in median survival time in human patients with GBM undergoing standard surgical resection/debulking with post-operative radiation therapy from 12 months to 15 months, and an increase in the two-year survival rate from $10 \%$ to $27 \%$ [15]. There have been no studies evaluating the efficacy of TMZ in canine patients with GBM, though systemic administration has been demonstrated as being tolerated in dogs receiving oral temozolomide at doses of $60-100 \mathrm{mg} / \mathrm{m}^{2}$ once daily for five days on a 28 day cycle, with gastrointestinal and hematologic effects most common [16,17]. In general, evaluation of chemotherapy for primary canine brain tumours is very limited; a recent study evaluating the use of lomustine (CCNU) for primary intracranial tumours in dogs revealed no survival benefit [2].

Despite this, the potential for the addition of chemotherapy to the treatment regimen of primary canine brain tumours remains attractive, especially if the risk for systemic side effects could be minimized or eliminated by using a sustained-release local implant approach. Local chemotherapy has been attempted using drugimpregnated implants in humans, and includes carmustine (Gliadel) wafers, and 5-fluorouracil poly(lactide-co-glycolide) (PLGA) microspheres [18-25]. However, results of these treatments have not been an improvement to standard of care. Additionally, carmustine 
wafers require surgical approach and resection of the tumour in order to allow placement [19]. PLGA can be combined with many drugs and molecules to allow extended release of the substance as the biopolymer breaks down and has demonstrated biocompatibility both in murine models and clinical trials in humans, including a trial involving 58 human patients that received PLGA combined with 5-fluorouracil without any demonstrated neurotoxicity [26]. PLGA's degradation properties are well-understood with an ability to adjust degradation time by altering the lactide to glycolide ratio [27-30] making it an attractive substrate, given its history of safety, for the delivery of local chemotherapy agents. In addition to its benefit in human GBM patients as an oral chemotherapeutic, TMZ has been described as an ideal candidate for local chemotherapy within the brain given its lack of need for hepatic activation, and its lack of neurotoxicity to normal brain tissue. TMZ rapidly hydrolyzes under normal and alkaline $\mathrm{pH}$, while it is stable at an acidic $\mathrm{pH}$, which is typical of an intra-tumoural environment; this allows TMZ to be taken up into tumour cells where its active metabolite is created, but reduces risk to normal tissues [31]. Because of the desirable properties of PLGA and TMZ and the proven benefit of oral TMZ for human GBM, these two agents were chosen for a local chemotherapy protocol that can be developed as a minimallyinvasive treatment option for primary canine brain tumours.

This type of drug delivery system can be used most effectively in patients if its detection is possible via advanced imaging after administration, as this will allow outcome measures to be associated with appropriate microcylinder placement and permit revision of implantation when indicated. Therefore, the safety of PLGA microcylinders combined with both TMZ and a gadolinium-based MR contrast agent was evaluated in this study. Our hypothesis for this study was that implantation of varying numbers of PLGA/TMZ microcylinders with different gadolinium concentrations would be neurologically tolerated in canines and that the lowest percentage of gadolinium will allow MRI visualization of the microcylinders.

\section{Methods}

Eight young adult, male intact, purpose-bred Beagle dogs were included in this study. All dogs were reportedly healthy prior to acquisition. Weights of dogs ranged between 7.7 and $10 \mathrm{~kg}$. A complete blood count, chemistry and urinalysis for all dogs was unremarkable and fecal examination revealed roundworms, for which all dogs were treated with fenbendazole at $50 \mathrm{mg} / \mathrm{kg}$ PO q24h for three days, which was repeated three weeks later. This study was approved by the University of Georgia Institutional Animal Care and Use Committee (IACUC).

Physical and neurologic examinations were performed by a single author $(\mathrm{JH})$ one day prior to the study period and then repeated 1,7 , 14, 21 and 28 days after microcylinder implantation in all dogs.

\section{Poly (lactide-co-glycolide) microcylinders}

Novel PLGA microcylinders measuring $5 \mathrm{~mm} \times 0.8 \mathrm{~mm}$ were created using a 50:50 molar ratio of DL-lactide to glycolide, which was designed for a thirty day degradation time. The microcylinders were made via melt extrusion using an injector and silicon tubing (Microspherix LLC, Boca Raton, FL). Each microcylinder weighed 4 $\mathrm{mg}$ and was either left blank, with no temozolomide or gadolinium, or was combined with $0.5 \mathrm{mg}$ of temozolomide and either $6.25,12.5$ or $25 \%$ gadolinium by weight. For the purpose of implantation, an 18 gauge, $15 \mathrm{~cm}$ brachytherapy needle with a blunt "pencil tip" and blunt obturator was created (Kobold Medical, Veradale, WA). All microcylinders were sterilized using ultraviolet radiation prior to implantation.

\section{Magnetic resonance imaging}

All dogs underwent a pre-surgical brain MRI for the purposes of planning implantation. Each dog had an intravenous cephalic catheter placed aseptically and was heavily sedated with butorphanol $0.2 \mathrm{mg} / \mathrm{kg}$ (Torbugesic $10 \mathrm{mg} / \mathrm{ml}$; Zoetis Animal Health, Auckland, New Zealand) and dexmedetomidine $10 \mathrm{mcg} / \mathrm{kg}$ (Dexdomitor $375 \mathrm{mcg} / \mathrm{m}^{2}$; Orion Corporation, Orion Corporation Finland) given intravenously. All dogs were monitored for heart rate, respiratory rate and oscillometric blood pressure during the MRI, for which they were placed in sternal recumbency. Transverse T2-weighted (T2w) images of the brain were acquired to allow for implantation planning (Siemens Tim Symphony $1.5 \mathrm{~T}$ whole-body scanner, extremity (knee) coil).

Following microcylinder implantation, all dogs returned to MRI under the same general anesthesia (see below). The post-operative imaging protocol consisted of T2w, T2w fluid attenuation inversion recovery (FLAIR), $\mathrm{T} 2^{\star}$-weighted $\left(\mathrm{T} 2^{\star}\right), \mathrm{T} 1 \mathrm{w}$ and 3 -D proton density (PD)-weighted Spatial and Chemical shift Encoded excitation (SPACE) series in the transverse plane. Transverse images were acquired as follows: T2w=TE $105 \mathrm{~ms}$, TR $3500 \mathrm{~ms}$, flip angle $150^{\circ}$, slice thickness $2.5 \mathrm{~mm}$; T2W FLAIR=TE $88 \mathrm{~ms}$, TR $10500 \mathrm{~ms}$, flip angle $180^{\circ}$, slice thickness $2.5 \mathrm{~mm}$; T2* ${ }^{\star}=$ TE $26 \mathrm{~ms}$, TR $1100 \mathrm{~ms}$, flip angle $20^{\circ}$, slice thickness $2.5 \mathrm{~mm}$; T1w=TE $24 \mathrm{~ms}$, TR $700 \mathrm{~ms}$, flip angle $150^{\circ}$, slice thickness $2.5 \mathrm{~mm}$; 3-D PD SPACE=TE $41 \mathrm{~ms}, 1000 \mathrm{~ms}$, flip angle $150^{\circ}$, slice thickness $0.5 \mathrm{~mm}$. An isotropic True Fast Imaging with Steady State Precession (TruFISP) was acquired in the sagittal plane with these parameters: TE $4 \mathrm{~ms}$, TR $10 \mathrm{~ms}$, flip angle $28^{\circ}$, slice thickness $0.5 \mathrm{~mm}$. Prior to and following the intravenous administration of gadoliniumbased MR contrast (gadopentetate dimeglumine $0.1 \mathrm{mmol} / \mathrm{kg}$ intravenously [Magnevist; Bayer Healthcare Pharmaceuticals, Whippany, NJ USA]), a dorsal plane, isotropic T1-weighted (T1w) Magnetization-Prepared Rapid Acquisition Gradient Echo (MPRAGE) series was acquired with these parameters: TE $3 \mathrm{~ms}$, TR 2300, flip angle $15^{\circ}$, slice thickness $0.6 \mathrm{~mm}$. All sequences were acquired without interslice spacing. Because the SPACE, TruFISP and MPRAGE were isotropic acquisitions, they could be reconstructed in any plane following the conclusion of the study. The T2w, FLAIR, T2* and preand post-contrast $\mathrm{T} 1 \mathrm{w}$ images were acquired as part of routine assessment of the brain. TruFISP was used to discriminate hemorrhage from the magnetic susceptibility of the microcylinders. SPACE was used to provide sub-millimeter slice thickness images with some graywhite matter definition.

At 28 days post-implantation, all dogs underwent MRI with the identical protocol that was used in the initial post-implantation MRI. The study was performed on each dog once heavily sedated as previous with dexmedetomidine at $10 \mathrm{mcg} / \mathrm{kg}$ and butorphanol at $0.2 \mathrm{mg} / \mathrm{kg}$ intravenously following aseptic placement of a cephalic intravenous catheter. All dogs were monitored for heart rate, respiratory rate and oscillometric blood pressure during the MRI. All MRI studies were evaluated by a single author $(\mathrm{SH})$ and evaluated for changes associated with microcylinder implantation.

\section{Microcylinder implantation}

Following pre-implantation MRI, propofol (PropoFlo $2810 \mathrm{mg} / \mathrm{ml}$; Abbott Laboratories, Abbott Park IL USA) was administered at 2-6 
Page 3 of 11

$\mathrm{mg} / \mathrm{kg}$ intravenously to effect, allowing endotracheal intubation; general anesthesia was maintained during the craniotomy procedure using isoflurane (IsoFlo; Abbott Laboratories, Abbott Park, IL, USA). Pre-medication was not necessary, as all dogs remained markedly sedate following the pre-surgical MRI. Heart rate, respiratory rate, temperature and blood pressure measurements were regularly monitored for all dogs during anesthesia. Dogs were maintained on 10 $\mathrm{ml} / \mathrm{kg} / \mathrm{hr}$ of lactated ringer's solution during both MRI and craniotomy. Fur was clipped in preparation for a frontoparietal craniotomy and the surgical site was aseptically prepared. Following routine exposure of the skull, a frontoparietal craniotomy was performed; a $2 \mathrm{~cm} \times 1 \mathrm{~cm}$ defect was created in the skull using a highspeed pneumatic drill to allow access to the left prosencephalon. The implantation side was chosen without preference, but was kept the same between all subjects, and the prosencephalic location was used due to its large parenchyhmal area and higher proportion of prosencephalic primary brain tumours. Microcylinders were placed freehand using the implantation needle. Although stereotactic navigation would be recommended for clinical use, it was not available at the time of this safety study, and was believed to be unnecessary given the goal of general neurologic safety evaluation. Microcylinders were implanted in three columns approximately $1 \mathrm{~cm}$ apart with an initial depth of approximately $3 \mathrm{~cm}$. Dogs $1-4$ received six microcylinders with two implanted in each column, while dogs 5-8 received twelve microcylinders with four implanted in each column. Dogs 1-4 received microcylinders with $6.25 \%, 12.5 \%, 25 \%$ and $0 \%$ gadolinium. Dogs 5 and 6 received microcylinders with $6.25 \%$ gadolinium while Dogs 7 and 8 received microcylinders with $12.5 \%$ gadolinium (Table 1). The surgical sites were closed routinely using polydioxanone (PDS II; Ethicon Endosurgery Inc, Cornelia, GA USA) suture material for the muscular fascia, subcutaneous and subcuticular tissues and nylon (Ethilon; Ethicon Endosurgery Inc, Cornelia, GA USA) suture material for the skin. Following recovery from anesthesia, all dogs were given buprenorphine hydrochloride $0.02 \mathrm{mg} / \mathrm{kg}$ intravenously for pain control (Buprenex; Reckitt Benckiser Healthcare (UK) Ltd., Hull England). Buprenorphine $0.02 \mathrm{mg} / \mathrm{kg}$ intramuscularly q8-12h was administered for approximately 24 hours following surgery, after which time tramadol (Tramadol Hydrochloride tablets; Amneal Pharmaceuticals, Paterson, NJ USA) at approximately 2-3 $\mathrm{mg} / \mathrm{kg}$ PO q12h was started and continued for ten days. Trimethoprim sulfa antibiotics (Sulfamethoxazole and Trimethoprim tablets USP; Qualitest, Huntsville, AL USA) were administered at a dose of $10-15 \mathrm{mg} / \mathrm{kg}$ PO q12h for fourteen days following surgery. No steroids or non-steroidal anti-inflammatory drugs were administered to any dogs during or immediately prior to the study period.

\section{Cerebrospinal fluid collection}

Prior to microcylinder implantation, while under general anesthesia maintained with isoflurane, $0.5-1 \mathrm{ml}$ of cerebrospinal fluid (CSF) was collected aseptically via a cerebellomedullary cisternal puncture for routine analysis of cell count, protein and cytology. CSF samples for the same analysis were taken on days 7 and 28 following surgical implantation of the microcylinders while each dog was briefly anesthetized using propofol $6 \mathrm{mg} / \mathrm{kg} \mathrm{IV}$.

\begin{tabular}{|l|l|l|l|}
\hline Dog & Microcylinders $\mathbf{( m c )}$ & \% Gadolinium/mc & Total temozolomide $(\mathbf{m g})$ \\
\hline 1 & 6 & 12.5 & 3 \\
\hline 2 & 6 & 25 & 3 \\
\hline
\end{tabular}

\begin{tabular}{|l|l|l|l|}
\hline 3 & 6 & 0 & 0 \\
\hline 4 & 6 & 6.25 & 3 \\
\hline 5 & 12 & 12.5 & 6 \\
\hline 6 & 12 & 6.25 & 6 \\
\hline 7 & 12 & 12.5 & 6 \\
\hline 8 & 12 & 6.25 & 6 \\
\hline
\end{tabular}

Table 1: Microcylinder details by dog.

\section{Gross and histopathological examination of brains}

Following the final MRI procedure, while each dog was still heavily sedated, pentobarbital sodium (Beuthanasia-D Special; Schering Plough Animal Health, Union, NJ USA) was delivered intravenously at a dose of $1.5 \mathrm{ml}$ per $10 \mathrm{lbs}$ for the purpose of humane euthanasia. Brains were removed within an hour of euthanasia and fixed by immersion in $10 \%$ buffered formalin. Serial transverse sections were made at 5-10 mm intervals; sections exhibiting gross changes, along with sections taken at the same level on the contralateral side, were embedded in paraffin and $4 \mu$ sections stained with hematoxylin and eosin (HE). One section from each dog containing microcylinder tract(s) was additionally stained for CD18 (microglia and macrophage marker), CD3 ( $\mathrm{T}$ cell marker), CD79 (B cell marker) and GFAP (astrocyte marker) via immunohistochemistry and with alcian blue/ Periodic Schiff reaction (AB/PAS). Additionally, PLGA microcylinders were embedded in paraffin, sectioned at $4 \mu$ and stained with $\mathrm{HE}$ and $\mathrm{AB} / \mathrm{PAS}$ to evaluate the microscopic appearance of the microcylinders.

A qualitative assessment of the lesion was made from the HE stained sections documenting the presence/absence of the microcylinder, contents of the space where the microcylinder(s) would have been located, and the surrounding inflammatory response along with the degree of hemorrhage. The mean lesion diameter of microcylinder tract, central cavitation plus surrounding inflammation, was calculated for each animal.

The nature and subjective quantity (percentage) of the various inflammatory cells were evaluated by $\mathrm{CD} 18, \mathrm{CD} 3$, and $\mathrm{Cd} 79$ immunohistochemical staining. GFAP and CD18 immunohistochemistry was used to give a qualitative and quantitative assessment of the perilesional astrocytic and microglial reaction, respectively. To quantify the glial reaction around the bead tracts, the linear distance from the peripheral edge of the inflammation surrounding the microcylinder tracts to where staining became normal was measured.

For immunohistochemistry, heat induced epitope retrieval was performed with Citra Plus solution buffer (CD18, CD3, GFAP; Biogenex, San Ramon, CA) or Target Retrieval Solution, pH 9 (CD79; Dako, Carpinteria, CA) followed by primary antibody (mouse anticanine CD18 at 1:50 [Peter Moore, Davis, CA]; rabbit anti-CD3 at 1:1000 [Dako]; mouse anti-CD67 1:200 [Dako]; mouse anti-GFAP 1:4000 [Biogenex]). Secondary biotinylated anti-mouse or anti-rabbit (Vector Laboratories, Burlingame, CA) as appropriate was applied followed by HRP linked streptavidin (4+, Biocare Medical, LLC, Concord, CA). The reaction was visualized with 3,3' diaminobenzidine (Dako) and the slides counterstained with hematoxylin. 
Citation: Hicks J, Platt SR, Holmes SP, Howerth E, Haley A, et al. (2016) Clinical, Imaging and Pathological Characteristics of Brain Implanted Polylactic Co-Glycolic Acid Polymers Conjugated with Temozolomide. J Veterinar Sci Techno 7: 325. doi:10.4172/2157-7579.1000325

Page 4 of 11

\section{Results}

All dogs successfully completed all aspects of the study. Neurologic abnormalities were not present in any dogs prior to microcylinder implantation and there were no significant physical examination abnormalities prior to the study period. Dog 4 was neurologically normal and dogs 1-3 and 6-8 had an absent menace in the right eye as their only abnormality one day following implantation. Dog 5 experienced a noticeable surgical complication; his left lateral ventricle was entered during the initial placement of the implantation needle as evidenced by cerebrospinal fluid exiting the needle prior to its adjustment. Implantation was completed after redirection of the implantation needle, but this dog appeared behaviorally blind in both eyes with an absent menace $\mathrm{OU}$ as the neurologic exam abnormalities one day following surgery. Mild scleral hemorrhage was also present in Dog 5. Otherwise, the only notable physical examination abnormalities were associated with the surgical sites in all dogs, none of which experienced complications. Dogs 1-3 and 6-8 were neurologically normal by the day seven examination following implantation and dog 5 gradually regained neurologic function over the course of the study, first regaining a menace response OS and appearing neurologically normal by the day 28 post-implantation examination.

Adverse events were uncommon during the study period. Dog 8 had a single episode of loose stool the day following implantation whereas $\operatorname{dog} 2$ had chronic, mild diarrhea both before and following implantation that continued throughout the study period. Dog 6 had a single episode of mucoid stool following implantation, was hyporexic for approximately five days following the procedure, but continued to drink and otherwise appeared normal with no vomiting or additional diarrhea. Several dogs had a transient decrease in appetite for less than 24 hours following anesthesia and implantation. Dog 5 had mild, intermittent diarrhea and two episodes of suspected hematuria immediately following anesthesia and implantation, which resolved without additional therapy. Dog 3 experienced cardiopulmonary arrest lasting less than one minute associated with propofol anesthesia for a cerebrospinal fluid tap on day 7 following implantation. This appeared to be due to overly fast administration of the drug. He regained spontaneous circulation immediately following the onset of cardiopulmonary resuscitation and recovered from the episode in less than five minutes with a normal physical and neurologic examination at that time. He experienced no further adverse events during the course of the study.

\section{Magnetic resonance imaging}

Although microcylinder conspicuity was poor in those lacking the gadolinium label, some could still be detected. For unlabeled microcylinders, the area of implantation was identified due to the alteration in the cerebral parenchyma and/or the tracts associated with microcylinder placement (Figure1 Ai-Av). In contrast, all of the gadolinium labeled microcylinders were easily identified immediately post-implantation (Figure2 Bi-Bvi), particularly on the T1w imaging series. They were characterized on the $\mathrm{T} 1 \mathrm{w}$ images by low signal intensity tubular structures with a thin surrounding halo of high signal localized to the tissue immediately in contact with the microcylinder. The width of the surrounding high signal halo increased with increasing gadolinium-labeling dose (Figure2 Biv-Bvi). A surrounding hyperintense halo was also seen in the T2w and PDw, but was thinner on the T2 and less discrete on the PDw. The isotropic T1w MPRAGE images were superior; in these images, each microcylinder could be distinguished from the adjacent microcylinder. Some fragmentation of the microcylinders was also detected post-implantation.

Minimal cerebral parenchymal changes were seen in association with the microcylinder placement. On the T2, PDw and FLAIR images, there was good distinction between the gray and white matter parenchyma to the level of the microcylinder halo. Most implantation procedures resulted in minimal hemorrhage ( $7 / 8 \mathrm{dogs})$, at least in the forms detectable immediately post-implantation with $\mathrm{T} 2{ }^{\star} \mathrm{w}$ images. The susceptibility artifact created by the microcylinder prevented detection of hemorrhage within a 2-3 mm radius. Most subjects had ventricular hemorrhage (7/8). Following contrast administration, no abnormal parenchymal and meningeal enhancement was present. In addition, the halo of high signal around the microcylinder did not increase in thickness in the post-contrast T1w images immediately post-implantation. This indicates maintenance of the blood brain barrier in the immediate vicinity of the microcylinder at that time point.

In the 28-day post-implantation images, the conspicuity of the microcylinders was more similar to that of unlabeled microcylinders (Figure3 $\mathrm{Ci}-\mathrm{Cv}$ ) because of the lack of surrounding high signal on the T1w images. Additionally, all suspected microcylinders were seen as fragments or had undergone breakdown into small round remnant pieces. In accordance with the latter, the size of the susceptibility artifact created was reduced and non-uniform. None of the microcylinders or fragments migrated over this period of time. Diffuse increased cerebral parenchymal hyperintensity on T2w, PDw and FLAIR images was seen in all the cerebral hemisphere in the area of the microcylinders at 28 days post-implantation. This signal change was focused in the white matter and in some cases the periventricular gray matter. A definitive association between cerebral parenchymal hyperintensity and microcylinder gadolinium dose was not identified, but the greatest increased signal, in general, was seen around the microcylinders in all dogs. Chronic hemorrhagic foci were seen in some implantation tracts and remained in the ventricles. Following contrast administration, only a thin halo of enhancement was seen around all microcylinders.

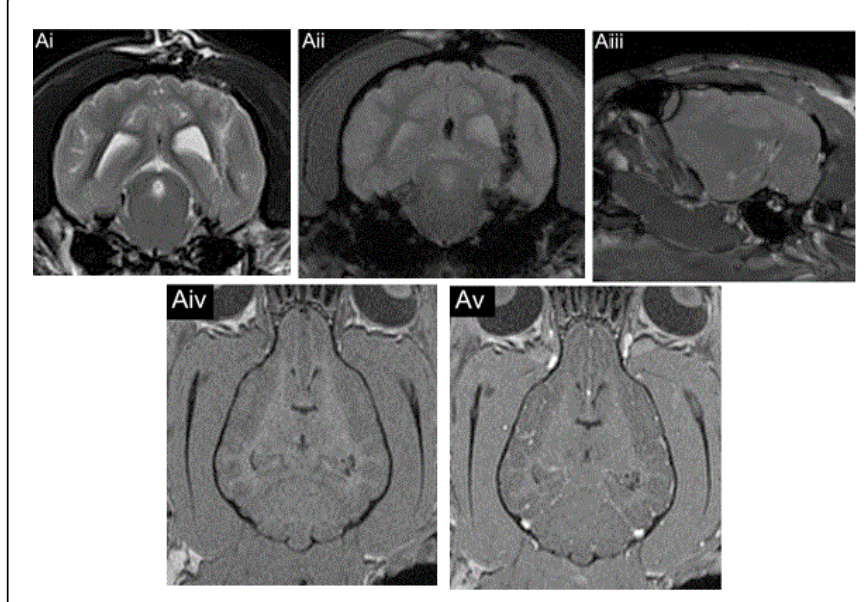

Figure1: (Ai) Unlabeled gadolinium microcylinders (MC) @24 h-T2 transverse, (Aii) $\mathrm{T}^{\star}$ transverse, (Aiii) TRUFI sagittal, (Aiv) T1 MPRAGE dorsal pre-IV contrast and (Av) post contrast. 
Citation: Hicks J, Platt SR, Holmes SP, Howerth E, Haley A, et al. (2016) Clinical, Imaging and Pathological Characteristics of Brain Implanted Polylactic Co-Glycolic Acid Polymers Conjugated with Temozolomide. J Veterinar Sci Techno 7: 325. doi:10.4172/2157-7579.1000325

Page 5 of 11

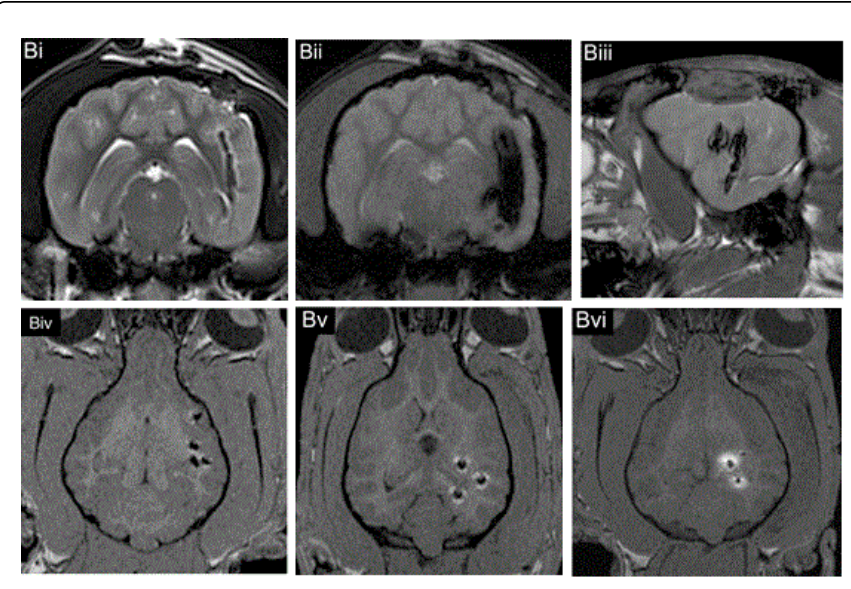

Figure 2: (Bi) Gadolinium-labeled MC @24 h post-implantation-T2 transverse, (Bii) $\mathrm{T}^{*}$ transverse, (Biii) TRUFI sagittal, (Biv) $\mathrm{T} 1$ MPRAGE dorsal with three doses of gadolinium labeled MC-6.25\%, (Bv) $12.5 \%$ and (Bvi) $25 \%$.
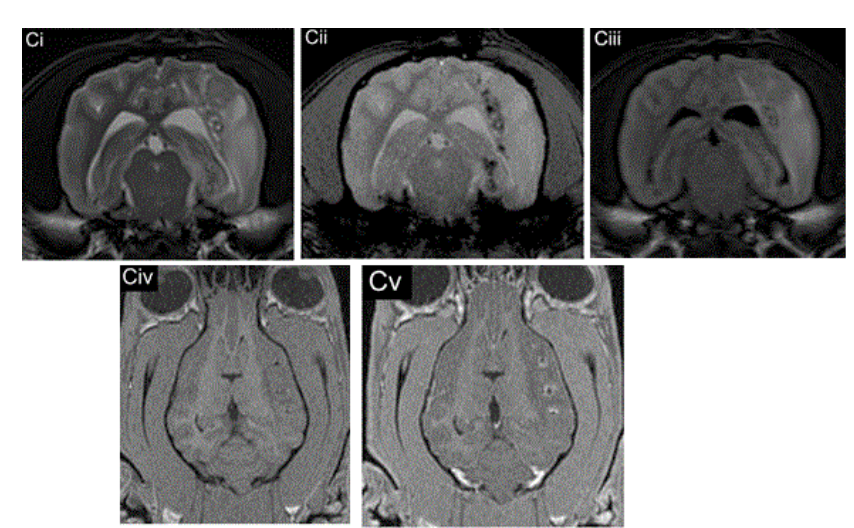

Figure 3: (Ci) Gadolinium-labeled MCG at 4 weeks following implantation-T2 transverse, (Cii) $\mathrm{T}^{\star}$ transverse, (Ciii) T2 FLAIR transverse, (Civ) T1w MPRAGE dorsal pre- and (Cv) post-IV contrast.

\section{Gross pathologic findings}

In all animals, a slight indentation of the dorsal cerebral surface corresponded to microcylinder tracts in the underlying neuroparenchyma. In transverse sections, there was mild dilation of the left, and sometimes right, lateral and third ventricles. Hemorrhagic tracts were observed in the cerebral hemisphere, extending into the internal capsule, thalamus, and sometimes the hippocampus. These tracts were more obvious in the animals that received 12 microcylinders (Figure 4). Intact microcylinders were not observed.

\section{Histopathologic findings}

Microscopically, the microcylinders were composed of parallel arrays of solid tubules that stained lightly basophilic on HE, did not stain with $\mathrm{AB} / \mathrm{PAS}$, and were birefringent at certain orientations (Figure 5 panel A). Such structures were not observed in any of the dog brains, suggesting that the microcylinders had completely broken down by the time of euthanasia.

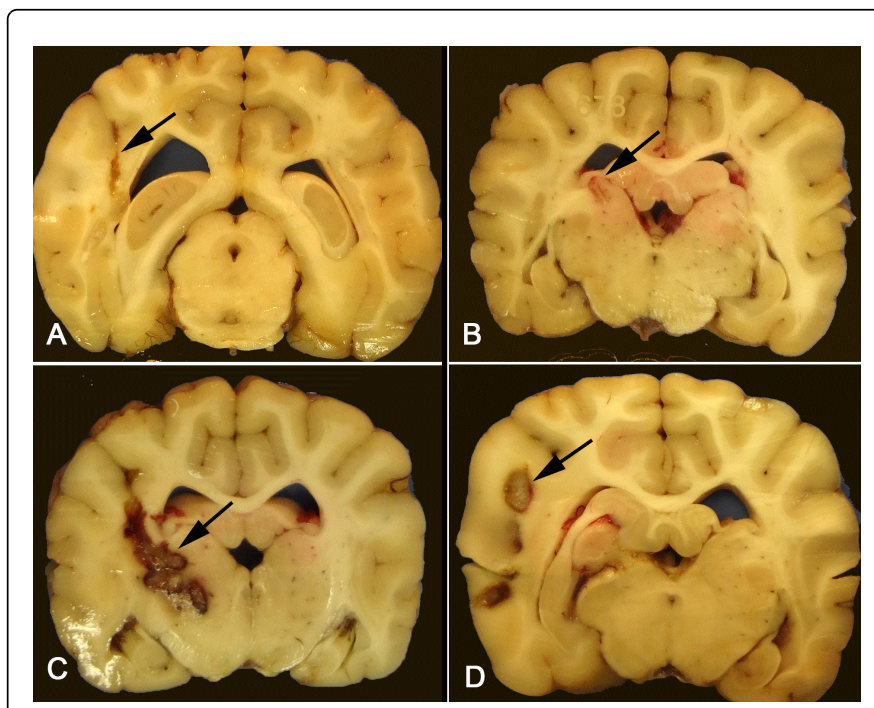

Figure 4: Transverse sections demonstrating typical gross lesions. Note dilation of lateral and third ventricles. A. Dog that received 6 untreated microcylinders has a fine linear hemorrhagic lesion in the cerebrum (arrow). B. Dog that received 6 microcylinders treated with temozolamide and $12.5 \%$ gadolinium has a slightly larger lesion (arrow). C. Dog receiving 12 microcylinders treated with temozolamide and $6.25 \%$ gadolinium has well defined hemorrhagic lesions larger in diameter than those in B. D. Dog receiving 12 microcylinders with temozolamide and $12.5 \%$ gadolinium has the largest diameter lesions (arrow), albeit not as striking as the coalescing lesions in $\mathrm{C}$.

Tracts where the microcylinders had been inserted were characterized by a central cavitation containing degenerating neutrophils and macrophages. The cavitations often contained amorphous intracellular basophilic material suspected to be phagocytosed microcylinder, and amorphous granular material, also suspected to be remnants of microcylinders. Sometimes hemorrhage, fibrin, or serous fluid was also present (Figure 5 panels B, C, D, E). This was surrounded by mild to severe granulomatous inflammation composed predominantly of epithelioid macrophages with variable numbers of multinucleated giant cells, fewer neutrophils, and some lymphocytes (Figure 5panel F). Peripheral to this was a thin layer of lymphocytes and plasma cells and, in some cases, mild to severe hemorrhage. Reactive vessels with variably thick cuffs of macrophages, lymphocytes and plasma cells were present in the surrounding neuroparenchyma. Extracellular lipid vacuoles were occasionally seen in the tracts and in the granulomatous reaction; epithelioid macrophages often had foamy cytoplasm and multinucleated cells often had discrete lipid vacuoles (Figure 5 panel G). The mean lesion diameter (cavitation plus inflammation) was widest in animals where 12 microcylinders had been inserted (mean lesion diameter range $1440.49 \mu-2776.85 \mu$ for 12 vs. mean lesion diameter range $824.95 \mu$ $-1556.36 \mu$ for 6$)$. The largest lesions were in dogs that received 12 microcylinders with $12.5 \%$ gadolinium and the smallest in the dog that received 6 microcylinders with 25\% gadolinium (mean lesion diameter $824.95 \mu$ ). The animal that received 6 untreated microcylinders had 
Citation: Hicks J, Platt SR, Holmes SP, Howerth E, Haley A, et al. (2016) Clinical, Imaging and Pathological Characteristics of Brain Implanted Polylactic Co-Glycolic Acid Polymers Conjugated with Temozolomide. J Veterinar Sci Techno 7: 325. doi:10.4172/2157-7579.1000325

Page 6 of 11

lesions of similar size to the dog receiving 6 treated with $0.5 \mathrm{mg}$ temozolamide and $6.25 \%$ gadolinium.

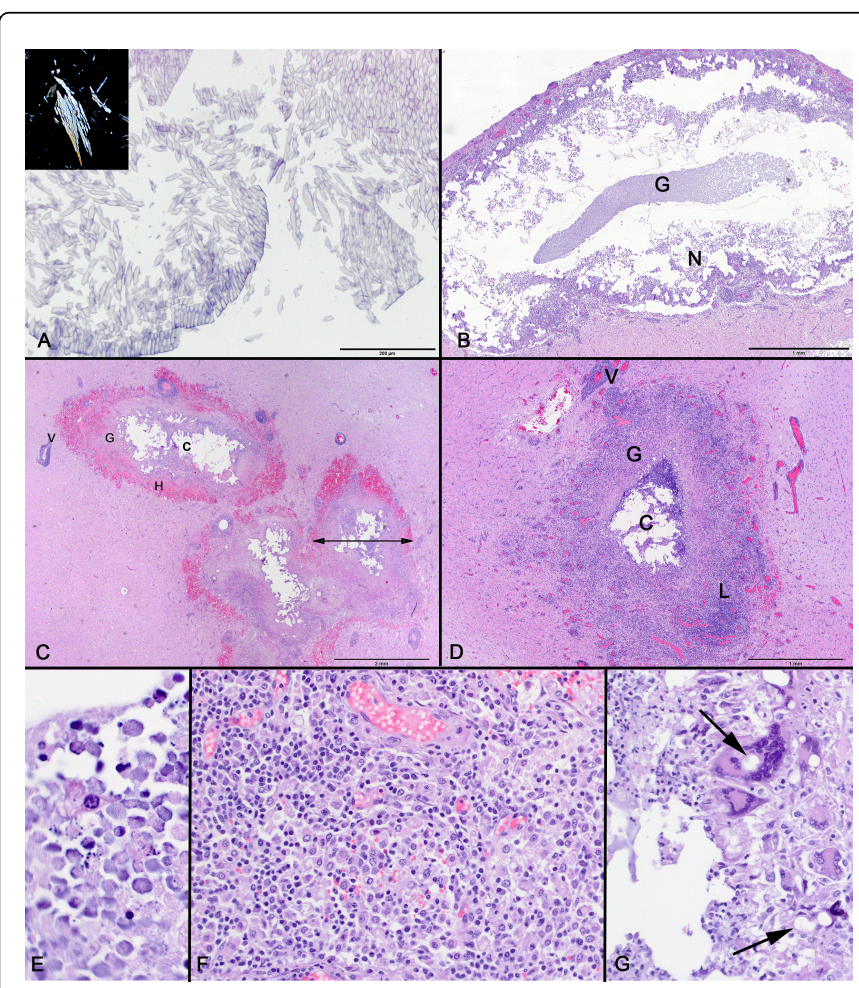

Figure 5: A. Microcylinders stained with HE are composed of parallel arrays of tubules that are birefringent (inset). B. Control dog microcylinder induced lesion has a large cavitation that contains a granular cast $(\mathrm{G})$ thought to be degraded remnants of a microcylinders and degenerating neutrophils (N). C. Dog that received 12 microcylinders with temozolamide and $6.25 \%$ gadolinium with three cross-sections of microcylinder tracts. Each has a central cavitation $(\mathrm{C})$ that is surrounded by granulomatous inflammation $(\mathrm{G})$ and a rim of hemorrhage $(\mathrm{H})$. Vessels $(\mathrm{V})$ in the adjacent neuroparenchyma have perivascular lymphoid cuffs. The double-ended arrow illustrates the measurement made to determine mean lesion diameter. D. Dog that received 6 microcylinders with temozolamide and 6.25 gadolinium with typical central cavitation containing degenerating neutrophils and macrophages (C)surrounded by a thick rim of granulomatous inflammation (G) and a more peripheral layer of lymphocytic infiltration (L). Vessels (V) have perivascular lymphoid cuffs. E. Degenerating macrophages containing amorphous material in microcylinder tract; this is suspected to be phagocytosed microcylinder debris. F. Higher magnification of granulomatous inflammation from lesion in $\mathrm{D}$ composed of epithelioid macrophages admixed with fewer lymphocytes. G. The granulomatous inflammation around the tracts often contained multinucleated giant cells containing lipid vacuoles (arrows). HE staining.

On immunohistochemistry, the granulomatous inflammation surrounding the tracts was intensely CD18 positive and admixed with small numbers of lymphocytes, mostly $\mathrm{T}$ cells. Perivascular cuffs contained larger numbers of lymphocytes that were also mostly $\mathrm{T}$ cells. Overall, $75-99 \%$ of the infiltrating lymphocytes were T cells.
With GFAP staining, an astrocytic reaction- both astrocytosis (increased numbers of cells) and astrogliosis (plump cells with increased numbers of processes)- extended away from the inflammatory reaction for a variable distance (Figure 6). A strong microglial reaction, usually of a similar magnitude, was also observed with increased numbers of plump microglia having many processes. While glial changes were seen in all dogs, those in the dog receiving microcylinders without temozolamide and gadolinium were the least extensive (CD18- $185.45 \mu$ and GFAP $568.12 \mu$ vs. mean of all animals with treated microcylinders of CD18- $2806.35 \mu$ and GFAP $2806.35 \mu$ ).

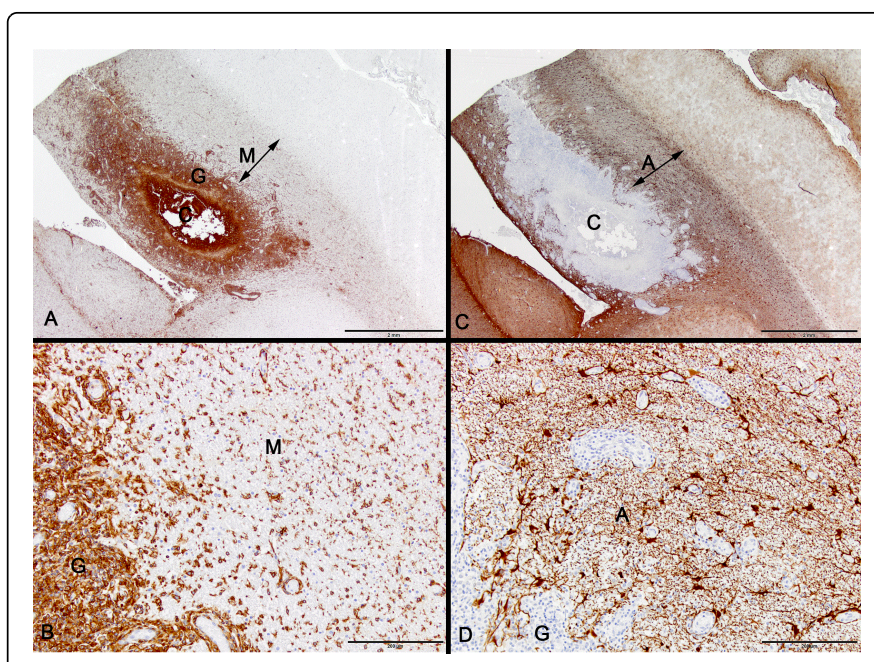

Figure 6: Immunohistochemistry for CD18 (A and B) and GFAP (C and D) for $\operatorname{dog} 679$ showing typical glial reaction. A. Intense staining for CD18 of the inflammatory cells in the microcylinder tract $(\mathrm{C})$ and surrounding granulomatous reaction $(\mathrm{G})$. A wide band of microgliosis $(\mathrm{M})$ the width of which is delineated by the double-headed arrow. B. Highermagnification showing an increased number of enlarged microglia ( $\mathrm{M}$; microgliosis) and intensely staining granulomatous inflammation $(\mathrm{G})$. C. GFAP does not stain the microcylinder tract (C) or surrounding granulomatous inflammation but there is a wide band of astrocytosis and astrogliosis around the microcylinder induced lesion (A) which is delineated by the double-headed arrow. D. Higher magnification of the astrocytic reaction around the unstained granulomatous inflammation $(G)$ showing increased number of astrocytes with plump cell bodies and numerous processes (A).

\section{Cerebrospinal fluid analysis}

Pre-implantation CSF was considered normal with a total nucleated cell count (TNCC) $<5 / \mu \mathrm{l}$, protein $<35 \mathrm{mg} / \mathrm{dl}$, and no significant cytologic abnormalities in $7 / 8$ dogs and abnormal due to a pleocytosis (TNCC $8 / \mu \mathrm{l}$ ) in dog 4. All dogs had $\leq 10$ red blood cells (RBCs) $/ \mu \mathrm{l}$ except for $\operatorname{dog} 4$, who had $265 \mathrm{RBCs} / \mu \mathrm{l}$, but protein concentration was normal at $7.7 \mathrm{mg} / \mathrm{dl}$.

One week post-implantation CSF was considered mildly abnormal in all dogs. Dogs 7 and 8 had TNCC $\leq 5 / \mu$, but a mild lymphocytic pleocytosis was suspected based on cytology in $\operatorname{dog} 7$, and slight xanthochromia with $2653 \mathrm{RBCs} / \mu \mathrm{l}$ was present in dog 8 . In the remaining dogs, there was a pleocytosis (TNCC range 6-28/ $\mu$ l) classified as mixed with lymphocytic predominance in dogs 1,2 and 6, mild lymphocytic in $\operatorname{dog} 3$ and neutrophilic in dogs 4 and 5. Protein 
Citation: Hicks J, Platt SR, Holmes SP, Howerth E, Haley A, et al. (2016) Clinical, Imaging and Pathological Characteristics of Brain Implanted Polylactic Co-Glycolic Acid Polymers Conjugated with Temozolomide. J Veterinar Sci Techno 7: 325. doi:10.4172/2157-7579.1000325

Page 7 of 11

was normal in all but dog 2 (range 15.2-47.4 mg/dl). Dog 5 was considered to have blood contamination (RBCs 3980/ $\mu$ l), but was still determined to have a neutrophilic pleocytosis. Overall, mean and median for all parameters included $14.9(14) \mathrm{WBC} / \mu \mathrm{l}, 849(20.5) \mathrm{RBC} /$ $\mu \mathrm{l}$ and 22.3 (18.6) $\mathrm{mg} / \mathrm{dl}$ protein.

CSF samples taken 28 days post-implantation were considered

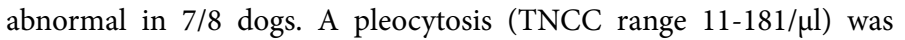

classified as lymphocytic in 5/7 dogs and as mixed pleocytosis with mononuclear or lymphocytic predominance in $2 / 7$ dogs. Overall, mean and median for all parameters included 73.9 (49) WBC/ $\mu \mathrm{l}, 29.3(5.5)$ $\mathrm{RBC} / \mu \mathrm{l}$ and 31.7 (27.4) $\mathrm{mg} / \mathrm{dl}$ protein (Table 2).

\begin{tabular}{|c|c|c|c|c|c|}
\hline \multicolumn{2}{|l|}{ Dog } & \multirow{2}{*}{ 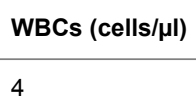 } & \multirow{2}{*}{$\begin{array}{l}\text { RBCs (cells/ } \mathbf{\mu l} \text { ) } \\
10\end{array}$} & \multirow{2}{*}{$\begin{array}{l}\text { Protein (mg/dl) } \\
8.3\end{array}$} & \multirow{2}{*}{$\begin{array}{l}\text { Cytology } \\
+ \text { +- increased neutrophils }\end{array}$} \\
\hline \multirow{3}{*}{1} & Pre & & & & \\
\hline & Day 7 & 21 & 3 & 17.2 & Mixed pleocytosis with lymphocytic predominance \\
\hline & Day 28 & 14 & 6 & 17.2 & Lymphocytic pleocytosis \\
\hline \multirow{3}{*}{2} & Pre & 1 & 5 & 8.4 & No cytologic abnormalities \\
\hline & Day 7 & 28 & 5 & 47.4 & Mixed pleocytosis with lymphocytic predominance \\
\hline & Day 28 & 11 & 0 & 15.4 & Lymphocytic pleocytosis \\
\hline \multirow{3}{*}{3} & Pre & 0 & 0 & 8.8 & No cytologic abnormalities \\
\hline & Day 7 & 7 & 108 & 15.2 & Mild lymphocytic pleocytosis \\
\hline & Day 28 & 105 & 22 & 43.4 & Mixed pleocytosis with mononuclear predominance \\
\hline \multirow{3}{*}{4} & Pre & 8 & 265 & 7.7 & Pleocytosis (acellular cytospin) \\
\hline & Day 7 & 25 & 1 & 19.9 & Neutrophilic pleocytosis \\
\hline & Day 28 & 50 & 188 & 20 & Lymphocytic pleocytosis \\
\hline \multirow{3}{*}{5} & Pre & 0 & 0 & 7.8 & No cytologic abnormalities \\
\hline & Day 7 & 26 & 3980 & 31.5 & Blood contamination; neutrophilic pleocytosis \\
\hline & Day 28 & 181 & 10 & 57.9 & Mixed pleocytosis with lymphocytic predominance \\
\hline \multirow{6}{*}{6} & Pre & 1 & 1 & 9.2 & No cytologic abnormalities \\
\hline & Day 7 & 6 & 19 & 22.8 & $\begin{array}{l}\text { Mild mixed cell pleocytosis with lymphocytic } \\
\text { predominance }\end{array}$ \\
\hline & Day 28 & 180 & 0 & 44.7 & Lymphocytic pleocytosis \\
\hline & Pre & 1 & 1 & 7.9 & No cytologic abnormalities \\
\hline & Day 7 & 5 & 22 & 12.7 & Possible mild lymphocytic pleocytosis \\
\hline & Day 28 & 39 & 3 & 26.4 & Lymphocytic pleocytosis \\
\hline \multirow[t]{3}{*}{8} & Pre & 1 & 0 & 10.1 & No cytologic abnormalities \\
\hline & Day 7 & 1 & 2653 & 11.6 & No cytologic abnormalities \\
\hline & Day 28 & 2 & 5 & 28.3 & No cytologic abnormalities \\
\hline \multirow{3}{*}{$\begin{array}{l}8 \\
\text { Mean (median) }\end{array}$} & Pre & $2(1)$ & $35.3(1)$ & $8.5(8.4)$ & $\mathrm{n} / \mathrm{a}$ \\
\hline & Day 7 & $14.9(14)$ & $849(20.5)$ & $22.3(18.6)$ & $\mathrm{n} / \mathrm{a}$ \\
\hline & Day 28 & $73.9(49)$ & $29.3(5.5)$ & $31.7(27.4)$ & $\mathrm{n} / \mathrm{a}$ \\
\hline
\end{tabular}

Table 2: Cerebrospinal fluid results.

\section{Discussion}

While primary brain tumours represent a relatively small percentage of overall cancer cases in dogs and people, their effects are typically devastating and surgical removal is often hampered by the difficulty in accessing some tumours without causing unacceptable damage to local tissues, nerves or blood supply. Additionally, the blood-brain barrier, composed of tight junctions between endothelial cells and foot 
processes of astrocytes that surround the cerebral microvasculature, is responsible for excluding the majority of substances, including many drugs, from entering the central nervous system (CNS) [32]. At least $98 \%$ of small molecules and essentially $100 \%$ of large molecules are excluded from the CNS by the BBB [18]. Intensive research is being performed to find new drugs and mechanisms for drug delivery across the BBB that will allow treatment of a variety of CNS disorders [33-35]. However, the possibility of bypassing the $\mathrm{BBB}$ entirely is extremely attractive, especially when considering a focal lesion that could be directly targeted, such as a brain tumour. And while lesions such as brain tumours have some disruption of the $\mathrm{BBB}$, this appears inadequate to allow effective use of most systemic chemotherapy. Temozolomide, a chemotherapeutic drug that is able to alkylate/ methylate DNA, is capable of crossing the blood-brain barrier but, in humans, the CSF concentration of the drug is only approximately $20 \%$ of the plasma concentration [36]. Despite this, orally-administered temozolomide is recommended as standard-of-care in a multi-modal approach to glioma therapy in humans [15]. If the drug can be delivered locally, higher concentrations may be reached in the tumour without causing systemic side effects, which could significantly improve the efficacy of temozolomide against susceptible gliomas. Gastrointestinal and myelo-toxicities are the most commonly reported adverse effects of temozolomide therapy and as many as $15 \%$ of human GBM patients have to discontinue systemic chemotherapy due to these effects in a recent study. The combined use of temozolomide and carmustine wafers, with surgical resection and radiotherapy, was also shown to increase the risk of these toxicities further [20]. Given that gastrointestinal or myelosuppressive adverse effects are more likely when a higher drug dose is administered, a proposed benefit of chemotherapy delivered directly to a lesion would be to lower the dose needed to achieve a positive clinical effect, which will secondarily reduce the risk of systemic side effects while maximizing the dose delivered to the tumour. Local chemotherapy can avoid the systemic dispersion and loss of chemotherapeutic agents into non-target tissues. In humans side effects can be significant and require dose reduction [37]. The use of Gliadel $^{\circledR}$ wafers placed into a resection cavity has been associated with cerebral edema, healing abnormalities of the surgical site, CSF leaks, intracranial infections, seizures, hydrocephalus and cyst formation [25], indicating the need to evaluate other local chemotherapy options. Additional benefits to this delivery modality utilizing PLGA microcylinders include being able to adjust the rate of administration to allow for long-term drug delivery with a single application, decreased invasiveness compared to surgical resection, and an improved cost-effectiveness with far fewer anesthetic episodes compared to conventional radiation therapy. In the future, other chemotherapeutic drugs that do not cross the BBB, but which may have even greater efficacy against primary brain tumours, can be evaluated using local delivery once a safe method has been established.

PLGA polymer implants have been widely evaluated in murine models and are considered biocompatible. The PLGA polymer degrades via hydrolysis and the material is ultimately degraded to water and carbon dioxide. There is a noted microglial-macrophagic reaction that occurs, but decreases dramatically by one month. In order to limit the number of dogs used and/or any added morbidity, histopathology was not performed at time points prior to study end in order to determine if inflammation was increasing or abating by one month. Additionally, given that the microcylinders were entirely broken down at the end of the study and no organisms were present to incite further inflammation, there is no reason to suspect that it might have increased following that time point. The polymer is non- neurotoxic as evidenced by normal neurons and axons amongst the inflammatory reaction, and there is no T lymphocyte reaction [27-30]. This is consistent with the finding of only mild inflammatory changes associated with the blank PLGA microcylinder in dog 3. The ratio of lactide to glycolide can be adjusted to alter the expected degradation time and allow for unique treatment plans based on the targeted disease and method of therapy, [19] though this would require evaluation of the conjugated drug dose needed for various release rates. For our study, microcylinders that are amenable to stereotactically-guided implantation were created, and have the potential to allow treatment of either surgically accessible or inaccessible tumours in a minimally-invasive manner. Stereotactic guidance systems have been validated for use in dogs previously using both CT and MRI [38-45].

Post-delivery imaging of local therapies for brain tumours in veterinary medicine has been evaluated using cetuximab conjugated to iron-oxide nanoparticles [46] and CPT-11 with a liposome carrier [47]. Gadolinium was chosen due to its previously successful combination with PLGA for post-implantation imaging, as discussed below, and its ready availability. It was combined successfully with the microcylinders in our study and provided good clinical imaging, even when used at a low percentage concentration. A gadolinium-based contrast agent has been previously incorporated into biodegradable microparticles that were imaged successfully in the mouse bladder, and, as in this study, the $\mathrm{T} 1$ effect was directly related to the level of gadolinium within the microparticles [48]. Gadolinium-impregnated PLGA microparticles have been successfully imaged as both a standard and modified polymer, which is consistent with our demonstration that gadoliniumPLGA microcylinders could be evaluated post-implantation using MRI [49-51]. Additionally, non-PLGA-based microparticles combined with gadolinium have been created and also appear to be suitable for use with MRI [52].

Microcylinder-associated changes were microscopically similar regardless of treatment or number inserted; however, changes were more severe in brains where 12 had been inserted and most severe in the brain where those microcylinders contained $12.5 \%$ gadolinium. Two dogs each were implanted with twelve microcylinders at 6.25 and $12.5 \%$ gadolinium. This implantation type was duplicated to help better evaluate toxicity where it was more expected given the large number of microcylinders. Additionally, 25\% gadolinium was not evaluated at the higher microcylinder load due to the concern for toxicity and the lesser likelihood that that percentage would be needed clinically. Lesions consisted of a central cavitation filled with inflammatory cells and disintegrated microcylinder material, surrounded by granulomatous and lymphocytic inflammation, and, sometimes, extensive hemorrhage. This finding is noteworthy as PLGA in murine models without other components has been thoroughly evaluated and a lymphocytic reaction is not reported. Given that a lymphocytic reaction was present even in the dog with blank microcylinders, the cause does not appear to be either temozolomide or gadolinium. It is unclear whether a difference in CNS immunology of the dog compared to a murine model, the large size of the microcylinders compared to previously-evaluated PLGA microspheres, our use of UV sterilization for the microcylinders or some other factor contributed to the T cell lymphocyte reaction seen in this study. The microcylinders had disintegrated into granular masses that appeared to then be phagocytosed by macrophages at 28 days post-implantation, which is consistent with their intended degradation speed at this formulation. 
Intravenous gadolinium-based formulations are hypertonic and known to cause local tissue reactions if extravasation occurs, as has been demonstrated in rat and mouse models. Higher osmolar agents such as gadopentetate dimeglumine and gadoversetamide appear to cause more severe reactions; [53] however, there is evidence that an intrinsic tissue toxicity of gadopentetate dimeglumine is present regardless of the high osmolarity, as demonstrated when that formulation was evaluated versus hypertonic saline in one study [54]. Interestingly, the inflammatory reaction in the latter study began to abate after approximately one month, so a reduction in inflammation may have been seen in our dogs if they had been evaluated at a time later than the termination of our study. Tissue exposure to gadoliniumbased contrast agents regularly occurs in the brain within lesions that contrast-enhance, as enhancement indicates disruption of the bloodbrain barrier. Because the areas of implantation in the dogs in our study did not undergo significant contrast enhancement immediately after surgery, it is unlikely that the blood-brain barrier was disrupted other than that associated with surgical trauma. Given this, it is unlikely that additional intravenous contrast agent contributed to the inflammatory reaction seen on histopathology.

Overall, microcylinder implantation was well-tolerated based on repeated physical and neurologic examinations in the live canine subjects. Only mild neurologic abnormalities were associated with implantation in 6/8 dogs, and no abnormalities were detected on examination in one subject. One dog had more significant postimplantation abnormalities; this was the only subject where the lateral ventricle was obviously entered during surgery, though after postimplantation MRI evaluation it was determined that left ventricular hemorrhage had occurred in $7 / 8$ subjects. The subject that was transiently blind post-operatively had accidental implantation into his thalamus, which did not occur in other dogs, but the tract did not involve the right side of the brain. Presence of ventricular hemorrhage did not appear to correlate with post-implantation neurologic changes; for example, the dog with the normal neurologic examination also had hemorrhage detected via MRI, while the dog that did not have hemorrhage had a right menace deficit. However, mild ventricular dilation, found in the majority of dogs, could have been caused by intraventricular hemorrhage leading to mild hydrocephalus, given that intraventricular hemorrhage in newborns is the most common cause of acquired hydrocephalus [55]. Because the neurologic abnormalities appeared immediately following implantation and did not persist or worsen, it is reasonable to assume that the neurologic deficits were due to surgical trauma rather than the inflammatory changes that were discovered at necropsy. Although it was decided that stereotactic navigation was not crucial for safety evaluation of the microcylinders, it is likely that some adverse events following surgery could have been avoided had it been available and utilized. There was no clear trend in the inflammatory changes of the cerebrospinal fluid in any group, although the two dogs with the highest level of pleocytosis at day 28 were both implanted with twelve rather than six microcylinders, their gadolinium concentrations were different. The results of physical and neurologic examinations following implantation indicate that despite histopathologic changes at necropsy, the treatment would have been judged safe in all living patients. However, histopathology results indicate that the lowest concentration of gadolinium that allows postimplantation imaging should be used as higher concentrations may be correlated with a more significant inflammatory reaction. It is also important to note that no anti-inflammatory medications were used in this study. Clinical brain tumour patients in both human and veterinary medicine frequently receive steroid therapy as a portion of their treatment and it is likely that steroids would attenuate some of the inflammatory reaction noted in our dogs. Given that even without the use of steroids the inflammation did not affect our dogs neurologically, was similar between the blank microcylinders and those with $6.25 \%$ gadolinium/temozolomide, and that the microcylinders would be implanted intratumourally, it is even more likely that clinical patients will tolerate the therapy. Additionally, inflammation is expected with most direct tumour treatments, including surgery, and an inflammatory reaction within a tumour could even be desirable in clinical patients.

Although there is a strong historical basis for the consideration of local sustained-release chemotherapy using PLGA, this safety study was not intended to evaluate the efficacy of this treatment. Given ethical considerations of canine studies and the rapid hydrolysis of temozolomide in a normal brain environment, no attempts were made to determine the radius or speed of drug distribution via multiple brain tissue samplings or sacrifice of dogs at various time-points, as the former would have negated evaluation for safety and the latter would have required more canine subjects. It would be possible to determine the radius of effective drug distribution within a tumor by evaluating tumor cells for methylation of DNA at the O6 position of guanine, which occurs due to TMZ's active metabolite. A naturally-occurring tumor within the canine brain would be the ideal substrate in which to perform this measurement, though some difficulty might still exist due to the resistance of certain glioma cells against TMZ's DNA methylation [56]. Additionally, given the slow-release formulation, evaluation of effective drug diffusion within a tumor will need to be evaluated at multiple time-points during PLGA degradation. Because canine clinical patients are not an appropriate test subject to make these determinations, options for obtaining this information include utilizing a rat glioma model or using clinical outcomes such as tumor response based on MRI and progression-free survival to help establish the most effective protocol. Unfortunately, the adequate intratumoural dosage of temozolomide is unknown in both humans and canines, and intratumoural tissue dynamics are significantly different than that of a normal brain, making any data regarding the release or concentration of temozolomide within normal tissues inapplicable.

Despite the use of chemotherapy in this safety study, typical systemic adverse events were not monitored for or categorized as being related to local TMZ therapy. The lowest reported clinically-evaluated dose of TMZ in dogs is $60 \mathrm{mg} / \mathrm{m}^{2}$, given once daily for 5 days. The highest local TMZ dose used in our study was $6 \mathrm{mg}$, which is approximately $20 \mathrm{x}$ less than the systemic doses in previous studies, making the risk for systemic adverse events negligible.

\section{Conclusion}

Our results indicate that it would be appropriate to evaluate the potential clinical efficacy of temozolomide-and gadolinium-conjugated PLGA microcylinders as an intratumoural chemotherapy option for the treatment of primary canine brain tumours, but that the lowest concentration of gadolinium that allows for post-implantation imaging and the lowest number of microcylinders possible for tumour coverage should be used in clinical patients.

\section{Competing Interests}

PLGA microcylinders and a portion of the funding for this study were provided by Microspherix LLC, for which Dr. Ed Kaplan is founder and CEO. 


\section{Author's Contributions}

All authors participated in study design and/or critical evaluation of the study. JH and SP carried out all aspects of the study. JH prepared the manuscript with contributions regarding MRI results from $\mathrm{SH}$ and those regarding histopathology from EH. EK and JK designed and produced the PLGA microcylinders. All authors participated in editing and final approval of the manuscript, with particular contribution from SP and EK.

\section{Acknowledgements}

Thank you for the hard work and dedication by our research technicians, Lisa Reno, RVT and Ethan Karstedt, RVT. Thank you to Dr. Ed Kaplan and his company, Microspherix LLC, for providing a portion of the funding and the microcylinders for this study. Dr. Kaplan was involved in the design of this study, but Microspherix LLC and/or Dr. Kaplan was not directly involved in the decision to publish results.

\section{References}

1. Dickinson PJ (2014) Advances in diagnostic and treatment modalities for intracranial tumors. J Vet Intern Med 28: 1165-1185.

2. Van Meervenne S, Verhoeven PS, de Vos J, Gielen IMVL, Polis I, Ham, at al. (2014) Comparison between symptomatic treatment and lomustine supplementation in 71 dogs with intracranial, space-occupying lesions. Vet Comp Oncol 12: 67-77.

3. Snyder JM, Shofer FS, Van Winkle TJ, Massicotte C (2006) Canine intracranial primary neoplasia: 173 cases (1986-2003). J Vet Intern Med 20: 669-675.

4. Sturges B, Dickinson PJ, Bollen AW, Koblik PD, Kass PH, et al. (2008) Magnetic resonance imaging and histological classification of intracranial meningiomas in 112 dogs. JVIM 22: 586-595.

5. Johnson GC, Coates JR, Wininger F (2014) Diagnostic immunohistochemistry of canine and feline intracalvarial tumors in the age of brain biopsies. Veterinary Pathology 51: 146-160.

6. Niebauer GW, Dayrell-Hart BL, Speciale J (1991) Evaluation of craniotomy in dogs and cats. J Am Vet Med Assoc 198: 89-95.

7. Greco JJ, Aiken SA, Berg JM, Monette S, Bergman PJ (2006) Evaluation of intracranial meningioma resection with a surgical aspirator in dogs: 17 cases (1996-2004). J Am Vet Med Assoc 229: 394-400.

8. Klopp LS, Rao S (2009) Endoscopic-assisted intracranial tumor removal in dogs and cats: long-term outcome of 39 cases. J Vet Intern Med 23: 108-115.

9. Axlund TW, McGlasson ML, Smith AN (2002) Surgery alone or in combination with radiation therapy for treatment of intracranial meningiomas in dogs: 31 cases (1989-2002). J Am Vet Med Assoc 221: 1597-1600.

10. Mariani CL, Schubert TA, House RA, Wong MA, Hopkins AL, et al. (2015) Frameless stereotactic radiosurgery for the treatment of primary intracranial tumours in dogs. Vet Comp Oncol 13: 409-423.

11. Keller ET, Madewell BR (1992) Locations and types of neoplasms in immature dogs: 69 cases (1964-1989). J Am Vet Med Assoc 200: 1530-1532.

12. Lipsitz D, Higgins RJ, Kortz GD, Dickinson PJ, Bollen AW, et al. (2003) Glioblastoma multiforme: clinical findings, magnetic resonance imaging, and pathology in five dogs. Veterinary Pathology Online 40: 659-669.

13. Heidner GL, Kornegay JN, Page RL, Dodge RK, Thrall DE (1991) Analysis of survival in a retrospective study of 86 dogs with brain tumors. J Vet Intern Med 5: 219-226.

14. Omuro A, DeAngelis LM (2013) Glioblastoma and other malignant gliomas: a clinical review. JAMA 310: 1842-1850.
15. Stupp R, Hegi ME, Mason WP, van den Bent MJ, Taphoorn M JB, et al. (2009) Effects of radiotherapy with concomitant and adjuvant temozolomide versus radiotherapy alone on survival in glioblastoma in a randomised phase III study: 5-year analysis of the EORTC-NCIC trial. The lancet oncology 10: 459-466.

16. Cancedda S, Rohrer Bley C, Aresu L, Dacasto M, Leone VF, et al. (2014) Efficacy and side effects of radiation therapy in comparison with radiation therapy and temozolomide in the treatment of measurable canine malignant melanoma. Vet Comp Oncol.

17. Dervisis NG, Dominguez PA, Sarbu L, Newman RG, Cadile CD, et al. (2007) Efficacy of temozolomide or dacarbazine in combination with an anthracycline for rescue chemotherapy in dogs with lymphoma. J Am Vet Med Assoc 231: 563-569.

18. Gabathuler R (2009) Blood-brain barrier transport of drugs for the treatment of brain diseases. CNS Neurol Disord Drug Targets 8: 195-204.

19. Menei P, Montero-Menei C, Venier MC, Benoit JP (2005) Drug delivery into the brain using poly(lactide-co-glycolide) microspheres. Expert Opin Drug Deliv 2: 363-376.

20. Miglierini P, Bouchekoua M, Rousseau B, Dam Hieu P, Malhare JP, et al. (2012) Impact of the per-operatory application of GLIADEL wafers (BCNU, carmustine) in combination with temozolomide and radiotherapy in patients with glioblastoma multiforme: Efficacy and toxicity. Clinical Neurology and Neurosurgery 114: 1222-1225.

21. Lin SH, Kleinberg LR (2008) Carmustine wafers: localized delivery of chemotherapeutic agents in CNS malignancies. Expert Rev Anticancer Ther 8: 343-359.

22. Aoki T, Nishikawa R, Sugiyama K, Nonoguchi N, Kawabata N, et al. (2014) A Multicenter Phase I/II Study of the BCNU Implant (Gliadel Wafer) for Japanese Patients with Malignant Gliomas. Neurologia medico-chirurgica 54: 290-301.

23. Westphal M, Hilt DC, Bortey E, Delavault P, Olivares R, et al. (2003) A phase 3 trial of local chemotherapy with biodegradable carmustine (BCNU) wafers (Gliadel wafers) in patients with primary malignant glioma. Neuro Oncol 5: 79-88.

24. Nagpal S (2012) The role of BCNU polymer wafers (Gliadel) in the treatment of malignant glioma. Neurosurg Clin N Am 23: 289-295, ix.

25. De Bonis P, Anile C, Pompucci A, Fiorentino A, Balducci M, et al. (2012) Safety and efficacy of Gliadel wafers for newly diagnosed and recurrent glioblastoma. Acta Neurochir (Wien) 154: 1371-1378.

26. Menei P, Capelle L, Guyotat J, Fuentes S, Assaker R, et al. (2005) Local and sustained delivery of 5-fluorouracil from biodegradable microspheres for the radiosensitization of malignant glioma: a randomized phase II trial. Neurosurgery 56: 242-248.

27. Fournier E, Passirani C, Montero-Menei CN, Benoit JP (2003) Biocompatibility of implantable synthetic polymeric drug carriers: focus on brain biocompatibility. Biomaterials 24: 3311-3331.

28. Voges J, Lehrke R, Kim DG, Lucas C, Schroder R, et al (2002) Tissue reactions after long-term intracerebral implantation of three different types of biodegradable polylactide rods in the rat. J Exp Ther Oncol 2: 70-76.

29. Veziers J, Lesourd M, Jollivet C, Montero-Menei C, Benoit JP, et al. (2001) Analysis of brain biocompatibility of drug-releasing biodegradable microspheres by scanning and transmission electron microscopy. J Neurosurg 95: 489-494.

30. Emerich DF, Bruhn S, Chu Y, Kordower JH (1998) Cellular delivery of CNTF but not NT-4/5 prevents degeneration of striatal neurons in a rodent model of Huntington's disease. Cell Transplant 7: 213-225.

31. Brem S, Tyler B, Li K, Pradilla G, Legnani F, et al. (2007) Local delivery of temozolomide by biodegradable polymers is superior to oral administration in a rodent glioma model. Cancer Chemother Pharmacol 60: 643-650.

32. Huber JD, Egleton RD, Davis TP (2001) Molecular physiology and pathophysiology of tight junctions in the blood-brain barrier. Trends Neurosci 24: 719-725.

33. Ball K, Bouzom F, Scherrmann JM, Walther B, Decleves X (2013) Physiologically based pharmacokinetic modelling of drug penetration 
Citation: Hicks J, Platt SR, Holmes SP, Howerth E, Haley A, et al. (2016) Clinical, Imaging and Pathological Characteristics of Brain Implanted Polylactic Co-Glycolic Acid Polymers Conjugated with Temozolomide. J Veterinar Sci Techno 7: 325. doi:10.4172/2157-7579.1000325

Page 11 of 11

across the blood-brain barrier--towards a mechanistic IVIVE-based approach. AAPSJ 15: 913-932.

34. Passeleu-Le Bourdonnec C, Carrupt PA, Scherrmann JM, Martel S (2013) Methodologies to assess drug permeation through the blood-brain barrier for pharmaceutical research. Pharm Res 30: 2729-2756.

35. Pardridge WM (2012) Drug transport across the blood-brain barrier. J Cereb Blood Flow Metab 32: 1959-1972.

36. Ostermann S, Csajka C, Buclin T, Leyvraz S, Lejeune F, et al. (2004) Plasma and cerebrospinal fluid population pharmacokinetics of temozolomide in malignant glioma patients. Clin Cancer Res 10: 3728-3736.

37. Saito K, Mukasa A, Narita Y, Tabei Y, Shinoura N, et al. (2014) Toxicity and Outcome of Radiotherapy with Concomitant and Adjuvant Temozolomide in Elderly Patients with Glioblastoma: A Retrospective Study. Neurologia medico-chirurgica 54: 272-279.

38. Chen AV, Wininger FA, Frey S, Comeau RM, Bagley RS, et al. (2012) Description and validation of a magnetic resonance imaging-guided stereotactic brain biopsy device in the dog. Vet Radiol Ultrasound 53: 150-156.

39. Flegel T, Oevermann A, Oechtering G, Matiasek K (2012) Diagnostic yield and adverse effects of MRI-guided free-hand brain biopsies through a mini-burr hole in dogs with encephalitis. J Vet Intern Med 26: 969-976.

40. Giroux A, Jones JC, Bøhn JH, Duncan RB, Waldron DR, et al. (2002) A new device for stereotactic CT-guided biopsy of the canine brain: design, construction, and needle placement accuracy. Vet Radiol Ultrasound 43: 229-236.

41. Koblik PD, LeCouteur RA, Higgins RJ, Fick J, Kortz GD, et al. (1999) Modification and application of a Pelorus Mark III stereotactic system for CT-guided brain biopsy in 50 dogs. Vet Radiol Ultrasound 40: 424-433.

42. Moissonnier P, Blot S, Devauchelle P, Delisle F, Beuvon F, et al. (2002) Stereotactic CT-guided brain biopsy in the dog. J Small Anim Pract 43: 115-123.

43. Moissonnier P, Bordeau W, Delisle F, Devauchelle P (2000) Accuracy testing of a new stereotactic CT -guided brain biopsy device in the dog. Res Vet Sci 68: 243-247.

44. Taylor AR, Cohen ND, Fletcher S, Griffin JF, Levine JM (2013) Application and machine accuracy of a new frameless computed tomography-guided stereotactic brain biopsy system in dogs. Vet Rad and Ultrasound 54: 332- 342.

45. Troxel MT, Vite CH (2008) CT-guided stereotactic brain biopsy using the Kopf stereotactic system. Vet Radiol Ultrasound 49: 438-443.
46. Platt S, Nduom E, Kent M, Freeman C, Machaidze R, et al (2012) Canine model of convection-enhanced delivery of cetuximab-conjugated ironoxide nanoparticles monitored with magnetic resonance imaging. Clin Neurosurg 59: 107-113.

47. Dickinson PJ, LeCouteur RA, Higgins RJ, Bringas JR, Larson RF, et al. (2010) Canine spontaneous glioma: a translational model system for convection-enhanced delivery. Neuro Oncol 12: 928-940.

48. Chen HH, Le Visage C, Qiu B, Du X, Ouwerkerk R, et al. (2005) MR imaging of biodegradable polymeric microparticles: a potential method of monitoring local drug delivery. Magn Reson Med 53: 614-620.

49. Sitharaman B, Van Der Zande M, Ananta JS, Shi XF, Veltien A, et al. (2010) Magnetic resonance imaging studies on gadonanotube-reinforced biodegradable polymer nanocomposites. J of Biomedical Materials Research Part A 93: 1454-1462.

50. Zhang Y, Zhou J, Guo D, Ao M, Zheng Y, et al. (2013) Preparation and characterization of gadolinium-loaded PLGA particles surface modified with RGDS for the detection of thrombus. Int J Nanomedicine 8: 3745-3756.

51. Reynolds CH, Annan N (2000) Gadolinium-Loaded Nanoparticles: New Contrast Agents for Magnetic Resonance Imaging. J of the American Chemical Society 122: 8940-8945.

52. Zhang HW, Wang LQ, Xiang QF, Zhong Q, Chen LM, et al. (2014) Specific lipase-responsive polymer-coated gadolinium nanoparticles for MR imaging of early acute pancreatitis. Biomaterials 35: 356-67.

53. Runge VM, Dickey KM, Williams NM, Peng XJ (2002) Local Tissue Toxicity in Response to Extravascular Extravasation of Magnetic Resonance Contrast Media. Investigative Radiology 37: 393-398.

54. McAlister WH, McAlister VI, Kissane JM (1990) The effect of Gddimeglumine on subcutaneous tissues: a study with rats. AJNR Am J Neuroradiol 11: 325-327.

55. Romero L, Ros B, Rius F, Gonzalez L, Medina JM, et al. (2014) Ventriculoperitoneal shunt as a primary neurosurgical procedure in newborn posthemorrhagic hydrocephalus: report of a series of 47 shunted patients. Childs Nerv Sys 30: 91-97.

56. Perazzoli G, Prados J, Ortiz R, Caba O, Cabeza L, et al. (2015) Temozolomide Resistance in Glioblastoma Cell Lines: Implication of MGMT, MMR, P-Glycoprotein and CD133 Expression. PLoS One 10: e0140131. 